\title{
Super-Nernstian ion sensitive field-effect transistor exploiting charge screening in $\mathrm{WSe}_{2} / \mathrm{MoS}_{2}$ heterostructure
}

\author{
Sooraj Sanjay (iD), Mainul Hossain $\mathbb{D}^{2}{ }^{2}$, Ankit Rao ${ }^{1}$ and Navakanta Bhat $\mathbb{D}^{1 凶}$
}

Ion-sensitive field-effect transistors (ISFETs) have gained a lot of attention in recent times as compact, low-cost biosensors with fast response time and label-free detection. Dual gate ISFETs have been shown to enhance detection sensitivity beyond the Nernst limit of $59 \mathrm{mV} \mathrm{pH}^{-1}$ when the back gate dielectric is much thicker than the top dielectric. However, the thicker back-dielectric limits its application for ultrascaled point-of-care devices. In this work, we introduce and demonstrate a pH sensor, with WSe (top)/ $^{2}$ $\mathrm{MoS}_{2}$ (bottom) heterostructure based double gated ISFET. The proposed device is capable of surpassing the Nernst detection limit and uses thin high-k hafnium oxide as the gate oxide. The 2D atomic layered structure, combined with nanometer-thick top and bottom oxides, offers excellent scalability and linear response with a maximum sensitivity of $362 \mathrm{mV} \mathrm{pH}^{-1}$. We have also used technology computer-aided (TCAD) simulations to elucidate the underlying physics, namely back gate electric field screening through channel and interface charges due to the heterointerface. The proposed mechanism is independent of the dielectric thickness that makes miniaturization of these devices easier. We also demonstrate super-Nernstian behavior with the flipped $\mathrm{MoS}_{2}$ (top)/WSe (bottom) heterostructure ISFET. The results open up a new pathway of 2D heterostructure engineering as an excellent option for enhancing ISFET sensitivity beyond the Nernst limit, for the next-generation of label-free biosensors for singlemolecular detection and point-of-care diagnostics.

npj 2D Materials and Applications (2021)5:93; https://doi.org/10.1038/s41699-021-00273-6

\section{INTRODUCTION}

Measuring the ionic content or $\mathrm{pH}$ of an analyte is critical for a wide variety of applications, ranging from medical diagnostics, early disease detection, and genome sequencing to industrial process control, homeland security, and environmental monitoring $^{1,2}$. Solid-state sensors, such as the ion-sensitive field-effect transistors (ISFETs), offer considerable benefits over state-of-theart electrochemiluminescence, electrochemical, and optical sensing platforms for point-of-care applications. ISFETs enable rapid, real-time, low-power, low-cost, and label-free electronic detection in a compact setup that is highly compatible with the standard complementary metal-oxide-semiconductor (CMOS) process ${ }^{3,4}$. In addition, ISFETs allow on-chip integration of sensor and measurement circuitry on the same substrate using monolithic integration $^{5}$. Unlike metal-oxide-semiconductor (MOS) field-effect transistors (FETs), the gate metal in ISFETs is removed and replaced by a fluid gate which consists of a reference electrode capacitively coupled to the oxide layer through an electrolyte solution ${ }^{6}$. The $\mathrm{pH}$ sensing is based on the modulation of surface potential and channel conductance, when hydrogen ions in the electrolyte combine with hydroxyl groups on the gate dielectric, causing a shift in the threshold voltage ${ }^{7}$. This shift in threshold voltage for a unit change in $\mathrm{pH}$ is defined as the voltage sensitivity of the $\mathrm{pH}$ sensor $^{8}$. For classical ISFET devices, the maximum achievable voltage sensitivity for $\mathrm{pH}$ sensing is $59 \mathrm{mV}$ $\mathrm{pH}^{-1}$ at $25^{\circ} \mathrm{C}$, known as the Nernst limit ${ }^{9}$. Enhancement of sensitivity, beyond the Nernst limit, is necessary to improve the resolution of the $\mathrm{pH}$ sensor, especially for detecting small $\mathrm{pH}$ changes in biological samples with ultralow concentrations. Several efforts have been made to achieve super-Nernstian sensitivity $\left(>59 \mathrm{mV} \mathrm{pH}^{-1}\right)$ in ISFETs. Recent studies have exploited the steep-subthreshold behavior in negative capacitance FETs ${ }^{10}$ and tunnel FETs ${ }^{11}$ to demonstrate ISFETs with sensitivities beyond the Nernst limit. A more common approach to enhance sensitivity uses capacitive amplification through asymmetric dual gate configurations ${ }^{9,12-16}$. Compared with classical single gate $\mathrm{pH}$ sensors, the combination of a fluid gate and back gate offers significant signal amplification, overcoming the charge screening effect caused by the counter ions in the electrolyte ${ }^{17}$. The threshold voltage shift is modulated by the top- $\left(C_{\text {top }}\right)$ and bottom- $\left(C_{\text {bottom }}\right)$ gate capacitances, allowing the sensitivity to be increased by simply adjusting the ratio, $C_{\text {top }} / C_{\text {bottom }}{ }^{18}$. A simple approach to achieve super-Nernst sensitivity is by increasing the thickness of bottom dielectric or changing the dielectric constant (making $C_{\text {bottom }}<C_{\text {top }}$ ). However, thicker dielectric limits miniaturization and restricts the use of these sensor devices in point-ofcare applications. Considering the ultimate aim of scaling down the device to nanoscale dimensions, an alternate method of enhancing sensitivity needs to be explored, which is independent of the thickness of the gate dielectric. This can be achieved by exploiting the interface traps and defects in order to modulate the device capacitance, without (necessarily) increasing its dimensions.

Several studies have reported ultrascaled FET-based biosensors using transition-metal dichalcogenide (TMDC)-based twodimensional (2D) materials, like molybdenum disulfide $\left(\mathrm{MoS}_{2}\right)$ or tungsten diselenide $\left(\mathrm{WSe}_{2}\right)$, as the channel material ${ }^{19-23}$. Compared with silicon, the atomically thin $2 \mathrm{D}$ materials offer desired scalability, good electrostatics, and reduced flicker noise, leading to highly sensitive nano biosensors with excellent signal-to-noise ratio (SNR) ${ }^{24}$. Recently, FETs with Van der Waals WSe ${ }_{2}$ (top)/ $\mathrm{MoS}_{2}$ (bottom) vertical heterojunction have been intensively investigated for their unique electrical properties such as bandto-band tunneling, negative differential resistance, and ambipolar 


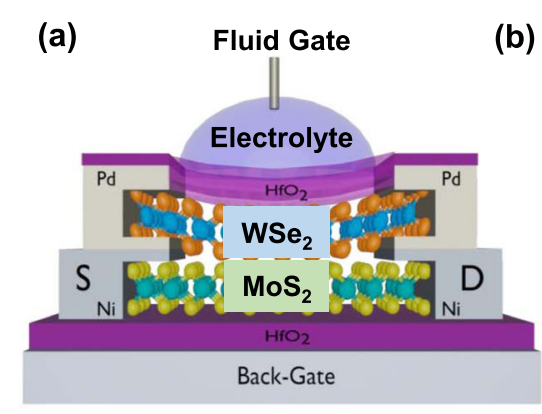

(d)

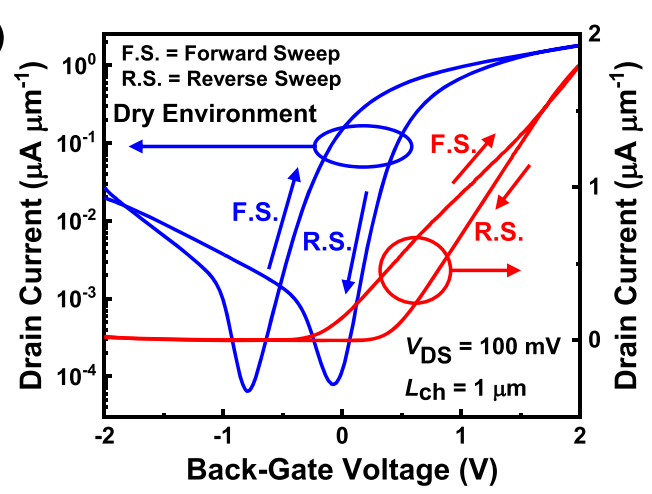

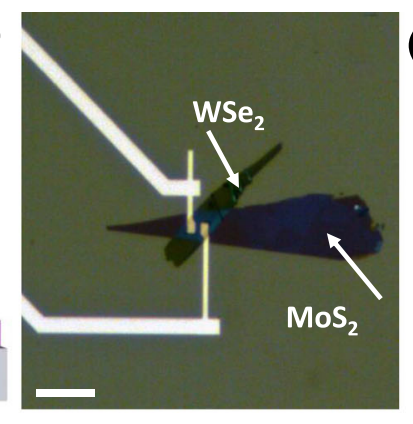

(c)

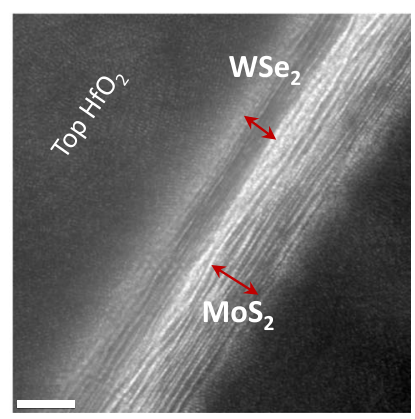

(e)

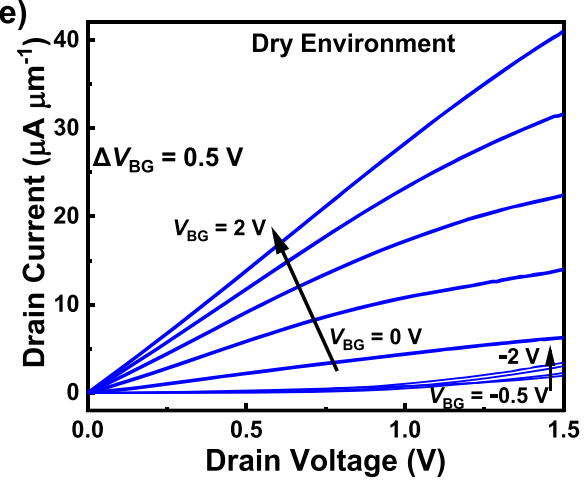

Fig. 1 Device structure and dry electrical characteristics. a Schematic of the WSe (top)/MoS $_{2}$ (bottom) ISFET device, b optical image, showing the $\mathrm{WSe}_{2}$ on $\mathrm{MoS}_{2}$ along with the metal contacts (scale bar $10 \mu \mathrm{m}$ ), c HR-TEM of the channel region of $\mathrm{HfO}_{2} / \mathrm{WSe}_{2}(\mathrm{top}) /$ $\mathrm{MoS}_{2}$ (bottom) $/ \mathrm{HfO}_{2}$ stack (scale bar $5 \mathrm{~nm}$ ), and $\mathbf{d}$ transfer and e output characteristics of the ISFET measured in the dry environment.

transfer characteristics, arising from the atomically sharp interface and strong interlayer coupling between the $\mathrm{MoS}_{2}$ and $\mathrm{WSe}_{2}$ films ${ }^{25-29}$. However, the use of TMDC 2D heterostructures has been limited to novel electronics, tunneling FETs, memory devices, photodetector, etc. In most of these applications, the nonidealities such as interface traps, defects, etc., deteriorate device performance. Alternatively, these nonideal effects can also be exploited and even engineered for sensing applications, as reported in recent literatures for super-Nernstian graphene-ISFETs ${ }^{30-32}$. In these devices, sensitivity above the theoretical Nernst limit is achieved through the process of charge-transfer doping, which directly modulates the carrier density of graphene with changes in $\mathrm{pH}$ level. However, this requires the fabrication of defectengineered nanocrystalline graphene grain boundaries, which may add to processing complexities. Here, we propose a WSe ${ }_{2}$ (top)/ $\mathrm{MoS}_{2}$ (bottom) $\mathrm{pH}$ sensor, which uses the interface effects of Van der Waals heterostructure to surpass the Nernst sensitivity limit of $59 \mathrm{mV} \mathrm{pH}^{-1}$ at room temperature. Unlike the defect-engineered graphene sensors, these still utilize an oxide to sense the $\mathrm{pH}$ by protonation or deprotonation. The sensing and signal transduction are decoupled using a double-gate FET architecture (fluid gate for sensing and back gate for gate voltage), while the charge screening due to the heterostructure interface is used to provide a gain over the Nernst limit.

In this work, we present experimental demonstration of a WSe ${ }_{2}$ (top)/ $\mathrm{MoS}_{2}$ (bottom) vertical heterostructure ISFET for $\mathrm{pH}$ sensing beyond the Nernst sensitivity limit. The double-gated FET structure uses a thin high- $\mathrm{k}$ hafnium oxide $\left(\mathrm{HfO}_{2}\right)$ as the top and bottom dielectric. The heterostructure enables the modulation of back gate transconductance by introducing a screening layer of interface and channel charges. This enables super-Nernst sensitivity by lowering effective back gate capacitance without increasing the device dimension and compromising the overall device performance. The atomically thin 2D heterostructure channel along with use of thin high-k dielectric allows extreme device scalability that is crucial for next generation nanobiosensors, targeted for point-of-care applications. In addition, we have also used technology computer-aided design (TCAD) simulations to elucidate the working principle of our device and also to investigate $\mathrm{pH}$ sensors with the flipped $\mathrm{MoS}_{2}$ (top)/ $\mathrm{WSe}_{2}$ (bottom) heterostructure as the channel material.

\section{RESULTS AND DISCUSSIONS}

\section{Device characterization}

Figure 1a shows the schematic of the ISFET device with the WSe $e_{2}$ (top)/ $/ \mathrm{MoS}_{2}$ (bottom) vertical heterostructure. Few-layer flakes of exfoliated $\mathrm{MoS}_{2}$ (bottom) and $\mathrm{WSe}_{2}$ (top) were stacked together and sandwiched between two $\sim 30 \mathrm{~nm}$ thick, $\mathrm{HfO}_{2}$ layers, serving as the top and the bottom gate dielectrics. The bottom $\mathrm{HfO}_{2}$ is deposited on a highly doped p-type silicon (Si) substrate, which also acts as a global back gate. Previous studies have reported $\mathrm{pH}$ sensors with $\mathrm{HfO}_{2}$ as the sensing surface due to the high $\mathrm{pH}$ sensitivity, low drift voltage, low hysteresis, and low body effect of $\mathrm{HfO}_{2}{ }^{33-35}$. Moreover, the medium permittivity and high bandgap of $\mathrm{HfO}_{2}$ provide excellent chemical stability when in contact with $\mathrm{Si}$ and silicon dioxide ${ }^{35}$. The $\mathrm{MoS}_{2}$ layer in the proposed device was contacted by nickel (Ni) electrodes and showed n-channel operation $^{36}$. Palladium (Pd) electrodes were used to make the contacts to the top $\mathrm{WSe}_{2}$ layer and shows p-channel (hole) transport ${ }^{37}$. The individual contacts on the flakes were also connected to form a single pair of source/drain contact. Figure $1 \mathrm{~b}$ shows the optical image of the $\mathrm{WSe}_{2}$ (top)/ $\mathrm{MoS}_{2}$ (bottom) heterostructure, with the source and drain metal contacts. Highresolution transmission electron microscope (HR-TEM) imaging was used to analyze the cross section of the fabricated device. The HR-TEM images shown in Fig. 1c show excellent interface quality with $\sim 1.2 \mathrm{~nm}$ Van der Waals gap between $\mathrm{HfO}_{2}-\mathrm{MoS}_{2}$ and $\mathrm{MoS}_{2}-\mathrm{WSe}_{2}$ interfaces. This gap is expected due to the dry transfer of the exfoliated flakes. There is minimal gap in 
WSe ${ }_{2}-\mathrm{HfO}_{2}$ interface, as the top $\mathrm{HfO}_{2}$ is deposited directly on WSe $e_{2}$. Both the top and bottom $\mathrm{HfO}_{2}$ dielectric are also of excellent quality without any visible cracks or nonuniformities. The same was also confirmed by atomic force microscopy (AFM) on the fabricated device (on the channel after top $\mathrm{HfO}_{2}$ ), which also showed good uniformity of deposition without any island formation (see Supplementary Fig. 1). The measured physical characteristics of the top and bottom $\mathrm{HfO}_{2}$ films are provided in Supplementary Table 1 . The top $\mathrm{HfO}_{2}$ was slightly thicker and showed a slight increase in roughness due to process variations. A more detailed physical and surface analysis of the same $\mathrm{HfO}_{2}$ films has been reported in the recent study by Ganapathi et al. ${ }^{38}$

The device was initially characterized in a dry environment by measuring the transfer and output characteristics as a function of back gate $\left(V_{\mathrm{BG}}\right)$ and drain voltage $\left(V_{\mathrm{DS}}\right)$, respectively. The transfer curve, in Fig. 1d, shows ambipolar characteristics, with $\mathrm{p}\left(V_{\mathrm{BG}} \downarrow\right.$ $\left.I_{\mathrm{D}} \uparrow\right)$ and $\mathrm{n}$ regions $\left(V_{\mathrm{BG}} \uparrow I_{\mathrm{D}} \uparrow\right)$ labeled. The transfer characteristics of the reverse sweep are considered to focus on the hole transport region. For $V_{\mathrm{BG}}<0 \mathrm{~V}$, the p-type $\mathrm{WSe}_{2}$ turns $\mathrm{ON}$ and starts conducting current through holes ( $p$-channel operation), whereas for $V_{\mathrm{BG}}>0 \mathrm{~V}$, $\mathrm{n}$-type $\mathrm{MoS}_{2}$ begins to conduct current by electrons (n-channel operation). The ON regions of these two materials are mutually exclusive, i.e., when one is $\mathrm{ON}$ and conducting, the other is fully depleted or even inverted. At large $V_{\mathrm{BG}}$, minority inversion carriers can form in one channel while the other is in accumulation. However, their contribution toward the overall device current is very small. The space charge regions at the $p-n$ junctions of the hetero-interface, as well as the corresponding band offsets, pose a significant potential barrier for the minority carriers to overcome. Furthermore, measurements on individual back-gated $\mathrm{MoS}_{2}$ and $\mathrm{WSe}_{2}$ FETs show minimal currents in inversion mode due to the large Schottky barrier at their respective contacts (see Supplementary Fig. 2). Also, the lack of any negative differential resistance (NDR) eliminates the possibility of band-to-band tunneling in our devices ${ }^{28}$. Our findings are consistent with the results reported by Lee et al. for a similar $\mathrm{WSe}_{2}$ (top)/ $\mathrm{MoS}_{2}$ (bottom) heterostructure ${ }^{39}$. The curves in Fig. 1e display the output characteristics of the proposed device at different $V_{\mathrm{BG}}$. The drain current increases with increasing $V_{\mathrm{BG}}$ during n-channel operation (from $V_{B G}=0$ to $2 \mathrm{~V}$ ), whereas it increases with decreasing $V_{\mathrm{BG}}$ for $\mathrm{p}$-channel operation (from $V_{\mathrm{BG}}$ $=-0.5$ to $-2 \mathrm{~V})$. The linearity of the curves indicates good ( ohmic) contacts with a low Schottky barrier.

The current in the $n$-channel region is $\sim 2$ orders higher than the $\mathrm{p}$-channel due to better back gate coupling for $\mathrm{MoS}_{2}$. The backgated transfer characteristics of the standalone $\mathrm{WSe}_{2}$ FET (Supplementary Fig. 2) show much higher currents, highlighting weaker back gate control in the $\mathrm{WSe}_{2}$ (top)/ $\mathrm{MoS}_{2}$ (bottom) heterostructure. The characteristics of the $n$-channel region are similar to that of a back-gated $\mathrm{MoS}_{2}$ FET, showing minimal effects of heterostructure on the bottom layer. The reverse is valid for topgate coupling, which allows the $\mathrm{WSe}_{2}$ layer to be better sensitive to $\mathrm{pH}$ changes during wet measurements. The bidirectional sweep of the $V_{\mathrm{BG}}$ reveals hysteresis in the device. The hysteresis direction confirms the lack of any ferroelectric polarization in the $\mathrm{HfO}_{2}$ films. The clockwise direction of the hysteresis arises from interface traps in the $\mathrm{HfO}_{2}-\mathrm{MoS}_{2}$ and $\mathrm{MoS}_{2}-\mathrm{WSe}_{2}$ interfaces ${ }^{40}$. These interface traps play a key role in reducing the back gate transconductance without the need for a thicker bottom gate dielectric. The density of these interface traps $\left(D_{\mathrm{it}}\right)$ is crucial for understanding the operation of the device. MOS capacitors are a popular way to extract interface characteristics. However, the small area of the exfoliated flakes (compared with the pads) prevents such measurements. The subthreshold slope (SS) can provide an approximation of $D_{\text {it }}$ (higher $D_{\text {it }}$ causes larger SS). But the complexity of the device structure and the transfer
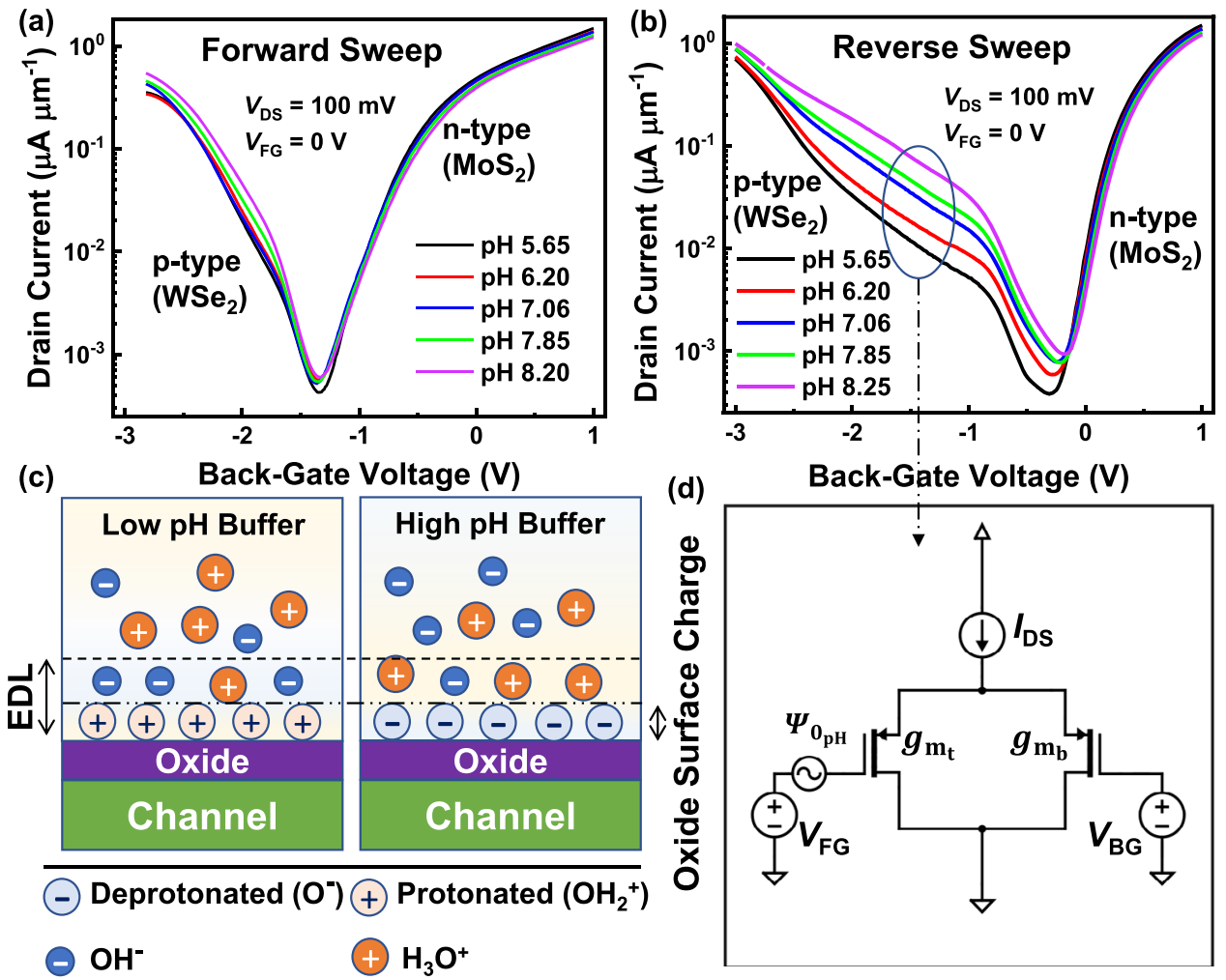

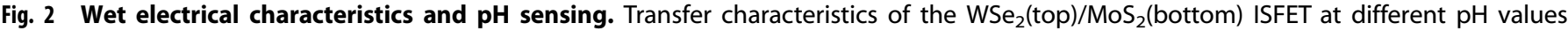
during a forward and $\mathbf{b}$ reverse sweep of the back gate voltage $\left(V_{\mathrm{BG}}\right)$, $\mathbf{c}$ schematic of the $\mathrm{pH}$ sensing mechanism showing the formation of surface charge on oxide and electric double layer (EDL) in the electrolyte, and $\mathbf{d}$ simplified equivalent model showing signal transduction in a double-gated device. 

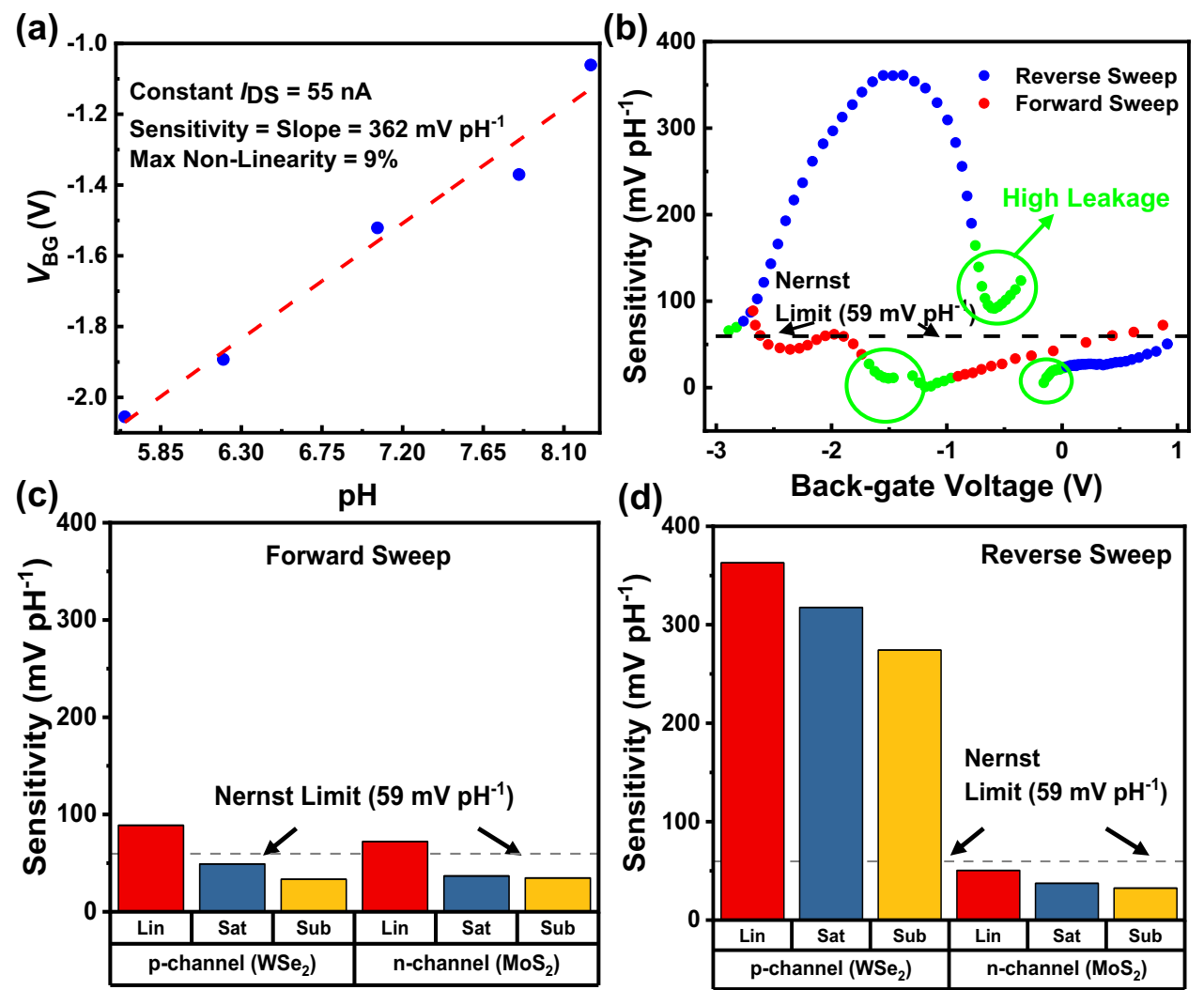

Fig. 3 Sensitivity. a Back-gate voltage $\left(V_{B G}\right)$ against $\mathrm{pH}$ at constant drain current, b Voltage sensitivity $\left(S_{\mathrm{V}}\right)$ with respect to the $V_{\mathrm{BG}}$ of the transfer curve at $\mathrm{pH}=7$. Peak $S_{V}$ at linear, saturation, and subthreshold region of operation during $\mathbf{c}$ forward sweep and $\mathbf{d}$ reverse sweep for

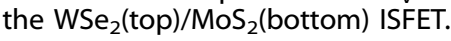

characteristics make such approximations difficult. Hence, an analysis based on TCAD simulations is provided, which numerically estimates a $D_{\text {it }}$ of more than $\sim 10^{12} \mathrm{~cm}^{-2}$ at the $\mathrm{MoS}_{2}-\mathrm{WSe}_{2}$ interface ${ }^{41}$.

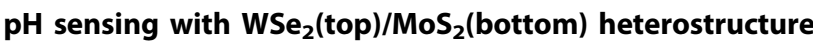 ISFET}

For the wet characterization and $\mathrm{pH}$ sensing, phosphate buffered saline (PBS) solutions of different $\mathrm{pH}$ values were prepared. These solutions were drop-casted onto the top $\mathrm{HfO}_{2}$ layer of the ISFET. A platinum wire, biased at $0 \mathrm{~V}$, was used as the reference electrode $\left(V_{\mathrm{FG}}\right)$. This reference electrode, in contact with the electrolyte solution, also serves as the fluid gate of the ISFET. The back gate transfer characteristics were obtained at multiple $\mathrm{pH}$ values, ranging from $\mathrm{pH}=5.65$ to 8.25 , in both forward- and reversesweep directions, as shown in Figs. $2 a$ and $2 b$, respectively.

The effective surface potential of the top dielectric is determined by the combination of fluid gate voltage, applied through the reference electrode, and the surface charge, modulated by the $\mathrm{pH}$ value of the electrolyte. $\mathrm{A}$ lower $\mathrm{pH}$ value results in protonation $\left(\mathrm{OH}+\mathrm{H}^{+}=\mathrm{OH}_{2}{ }^{+}\right)$, giving rise to positive surface charges, while at higher $\mathrm{pH}$ values, the top $\mathrm{HfO}_{2}$ surface is deprotonated $\left(\mathrm{OH}-\mathrm{H}^{+}=\mathrm{O}^{-}\right)$, yielding negative charges. These surface charges are partially screened by the counter-ions in the electrolyte, which move toward the charged surface and forms an electric double layer. The resultant surface charge or the change in potential at the oxide surface $\left(\Psi_{0_{p H}}\right)$ causes a corresponding shift in the device threshold voltage, which, in turn, determines the sensitivity of the $\mathrm{pH}$ sensor. A schematic of this sensing mechanism is shown in Fig. 2c.

Figure $2 \mathrm{~d}$ represents a simplified model of the signal transduction once a surface charge and potential have developed at the oxide surface. A dual gated configuration can be represented as two transistors in parallel with a common source and drain. The top gate with a transconductance $g_{\mathrm{m}_{\mathrm{t}}}$ acts as the sensor for the oxide surface potential $\Psi_{0_{p H}}$ (represented as an ac source) due to the $\mathrm{pH}$. The fluid gate bias $V_{\mathrm{FG}}$ is kept at a reference voltage of $0 \mathrm{~V}$. The device current is controlled by the back gate voltage $\left(V_{\mathrm{BG}}\right)$ and has a transconductance of $g_{\mathrm{mb}}$. When operated at constant current mode, the voltage sensitivity is given by Eq. 1. Hence, superNernstian sensitivity can be obtained if $g_{m_{b}}<g_{m_{t}}$ providing a gain in the sensitivity ${ }^{12,42,43}$.

$S_{V}=\frac{\Delta V_{\mathrm{BG}}}{\Delta \mathrm{pH}}=\left(\frac{g_{\mathrm{m}_{\mathrm{t}}}}{g_{\mathrm{m}_{\mathrm{b}}}}\right)\left(\frac{\Delta \Psi_{\mathrm{p}_{\mathrm{pH}}}}{\Delta \mathrm{pH}}\right)$

To measure the sensitivity of our device, we plot $V_{\mathrm{BG}}$ against the corresponding $\mathrm{pH}$ of the electrolyte solution at a fixed drain current $\left(I_{\mathrm{DS}}=55 \mathrm{nA}\right)$, as shown in Fig. 3a. The voltage sensitivity $\left(S_{v}\right)$ is derived directly from the slope and amounts to $362 \mathrm{mV}$ $\mathrm{pH}^{-1}$, clearly exceeding the Nernst limit by more than six times. The response, with a nonlinearity of $9 \%$, indicates excellent sensing performance. The $S_{\mathrm{V}}$ can be tuned by adjusting the $V_{\mathrm{BG}}$, as shown in Fig. $3 \mathrm{~b}$. Here, $S_{v}$ is determined at a constant drain current and plotted with respect to the $V_{\mathrm{BG}}$ of the transfer curve at $\mathrm{pH}=7$ for both forward and reverse sweeps. The peak $S_{v}$ in the subthreshold, linear, and saturation region of the WSe $\mathrm{W}_{2}$ (top)/ $\mathrm{MoS}_{2}$ (bottom) ISFET is displayed in Figs. $3 \mathrm{c}$ and $3 \mathrm{~d}$ for the forward and reverse sweeps, respectively. In the forward sweep, $S_{v}$ is high only in the extreme linear regions of both $p$ and $n$ sides. On the other hand, the $\mathrm{p}$-channel region in the reverse sweep shows high $S_{v}$ throughout subthreshold, linear, and saturation regions. In fact, the highest $S_{v}\left(362 \mathrm{mV} \mathrm{pH}^{-1}\right)$ is obtained in the reverse sweep when the back gate is biased in the linear region of hole transport. Although super-Nernstian sensitivity is visible in both the forward 
(a)
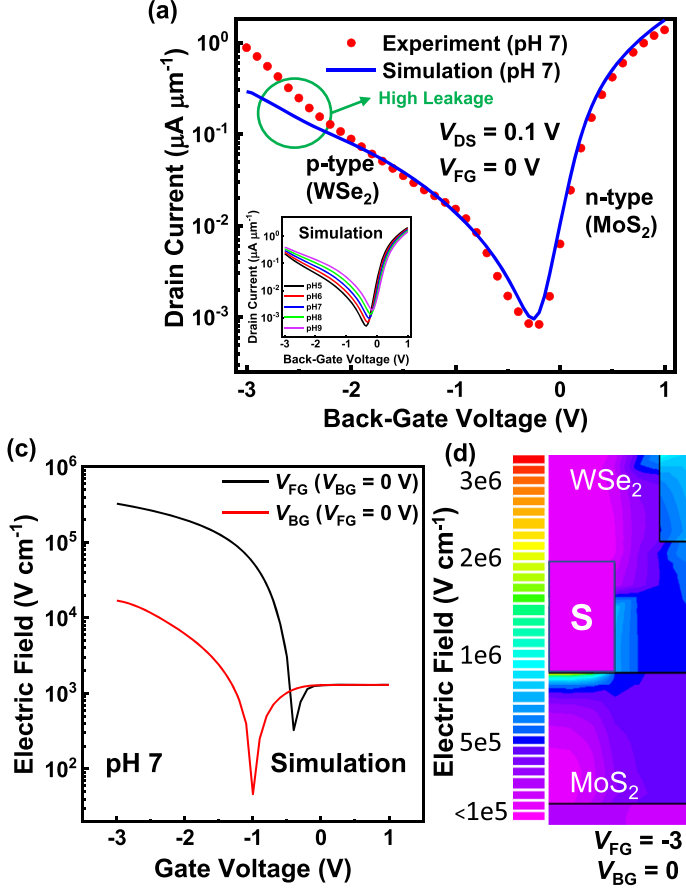

(b)

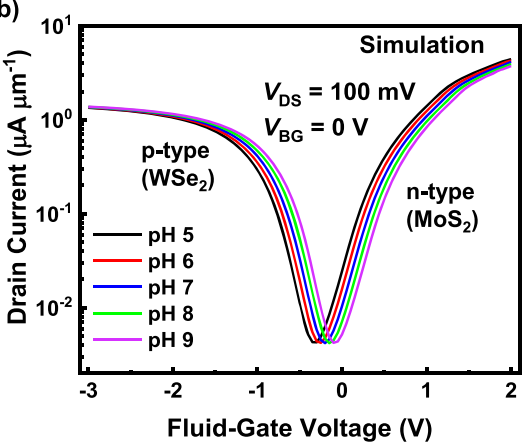

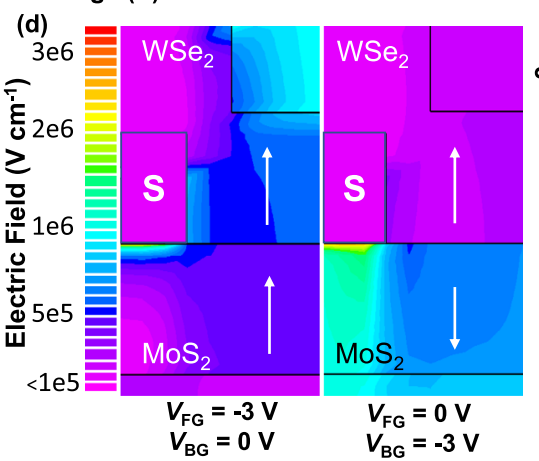

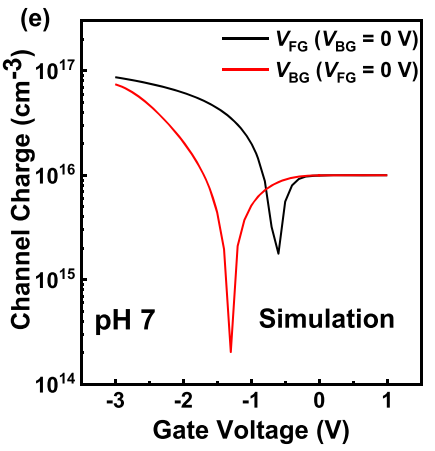

Fig. 4 TCAD simulations. a TCAD simulations calibrated against experimental transfer curve at pH $=7$ in the reverse-sweep direction for the

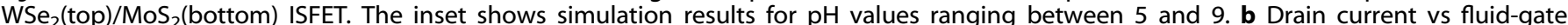
voltage characteristics at different $\mathrm{pH}$ values, extracted using TCAD simulations. c Simulated magnitude of the electric field across the WSe 2 channel v/s gate voltage (for $V_{\mathrm{FG}}$ and $V_{\mathrm{BG}}$ when the other is set to $0 \mathrm{~V}$ ). d Simulated electric field contours with applied $V_{\mathrm{FG}}$ and $V_{\mathrm{BG}}$ (arrows indicate the field direction). e Simulated density of channel (WSe $)_{2}$ charge $\mathrm{V} / \mathrm{s}$ gate voltage (for $V_{\mathrm{FG}}$ and $V_{\mathrm{BG}}$ when the other is set to $0 \mathrm{~V}$ ).

and the reverse sweeps, it is more prominent in the reverse sweep direction. The enhanced sensitivity, beyond the theoretical Nernst limit of $59 \mathrm{mV} \mathrm{pH}^{-1}$, originates from the transconductance $\left(g_{\mathrm{m}}\right)$ modulation between the fluid-gate and the back gate, since $g_{\mathrm{m}_{\mathrm{b}}}<g_{\mathrm{m}_{\mathrm{t}}}$.

In the present work, the $g_{\mathrm{m}}$ mismatch arises from the heterointerface properties, whereas it originates from capacitance mismatch in devices with a thicker back gate dielectric ${ }^{44}$. The donor traps in the $\mathrm{MoS}_{2}-\mathrm{WSe}_{2}$ interface capture holes from $\mathrm{WSe}_{2}$ during the reverse sweep and reduces $g_{m_{b}}$, leading to enhanced $S_{v}$. In addition, the combination of screening effect ${ }^{45}$ and mobility degradation ${ }^{46}$ increases $S_{v}$ in the linear region of operation. In the former case, the inversion charges in the bottom $\mathrm{MoS}_{2}$ channel lower the $g_{m_{b}}$ when the device is biased in strong linear region of the $\mathrm{p}$-channel side. In addition, high vertical electric field degrades the mobility of carriers through the channel through surface scattering, reducing $g_{\mathrm{m}_{\mathrm{b}}}$ and improving $S_{\mathrm{v}}$. A similar effect is observed in the strong linear region of the $n$-channel region during the forward sweep. In all cases, the lowest $S_{v}$ is observed in the subthreshold region, primarily due to the high gate leakage.

\section{Charge-screening effect for high sensitivity}

To further understand the role of screening and interface traps in enhancing $S_{\mathrm{v}}$, we investigate the electrical characteristics of WSe ${ }_{2}$ (top)/ $/ \mathrm{MoS}_{2}$ (bottom) heterostructure ISFET using TCAD. All simulations were carried out with Silvaco ATLAS device simulation tool, which self-consistently solves Poisson's equations along with current continuity equations to obtain the drain current as a function of gate voltage. Silvaco ATLAS TCAD device simulator tool cannot directly model devices based on 2D materials. As a workaround, we have modeled $\mathrm{MoS}_{2}$ and $\mathrm{WSe}_{2}$ using the material parameters available in the literature and considering the 3-D equivalent of their 2-D density of states. Using a few layers $(\sim 6 \mathrm{~nm})$ of exfoliated flakes means that the quantum effects dominant in monolayer or bilayer materials can be avoided. This approach is consistent with previous studies which used TCAD tools to simulate 2-D material based FETs, with reasonable accuracy ${ }^{41,47-49}$. The mobile ions in the electrolyte solution are modeled as charge carriers in a semiconductor material such that $\mathrm{pH}$ values correspond to the charge density at the electrolyte/ dielectric interface ${ }^{50}$. The use of $\mathrm{HfO}_{2}$ as the gate dielectric and sensing layer has been well explored in the literature and usually shows near-ideal sensitivity even in single gate configurations ${ }^{15,33,35}$. Hence, the modeling of surface protonation or deprotonation on the $\mathrm{HfO}_{2}$ surface is not included in the simulations. The TCAD model is calibrated against the drain current vs back gate voltage curve of $\mathrm{pH}=7$ in the reverse-sweep direction. Figure $4 a$ shows a good agreement between experiment and simulation. A slight deviation is observed at high $V_{\mathrm{BG}}$ due to gate-leakage in the practical device, which is not captured in the TCAD simulations. The inset of Fig. 4 a shows the simulation results for a wide range of $\mathrm{pH}$ values.

Figure $4 \mathrm{~b}$ shows the variation in the drain current with respect to the change in the fluid-gate voltage $\left(V_{\mathrm{FG}}\right)$ at different $\mathrm{pH}$ values, when $V_{\mathrm{BG}}=0 \mathrm{~V}$, as obtained from the TCAD simulations. The sensitivity with change in $V_{\mathrm{FG}}$ is found to be constant across all operating regions and is $\sim 59.5 \mathrm{mV} \mathrm{pH}^{-1}$, very close to the Nernst limit. This is expected as there is no gain mechanism with a single gated device. However, the device can still provide near-ideal sensitivity even when operated as a single gated ISFET.

The TCAD simulations confirm the presence of interface traps $\left(\sim 10^{12} \mathrm{~cm}^{-2}\right)$ at the $\mathrm{MoS}_{2}-\mathrm{WSe}_{2}$ interface as well as inversion carriers in the bottom $\mathrm{MoS}_{2}$ channel (Supplementary Fig. 3). The higher density of traps in $\mathrm{WSe}_{2}-\mathrm{MoS}_{2}$ interface and their distribution of energy levels have a profound impact on the device operation. The much lower currents and larger subthreshold slope of the p-channel region are due to these donorinterface traps that capture holes from the $\mathrm{WSe}_{2}$ channel as back gate bias is applied. On the contrary, the traps in the $\mathrm{HfO}_{2}-\mathrm{MoS}_{2}$ 

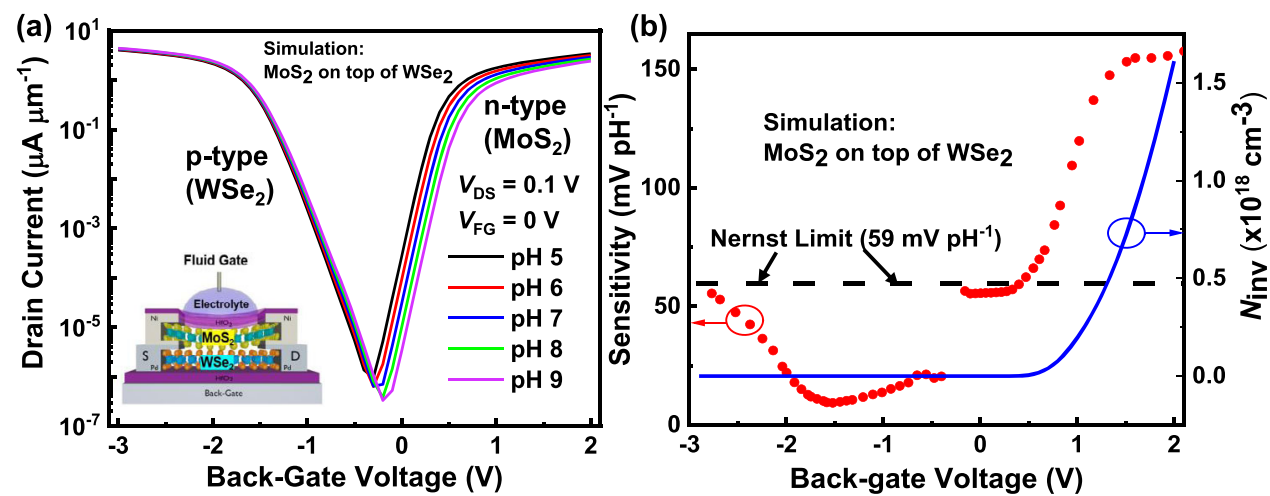

Fig. 5 Simulated $\mathbf{M o S}_{\mathbf{2}}$ (top)/WSe $\left(\right.$ bottom) ISFET. a Drain current vs back-gate voltage characteristics at different $\mathrm{pH}$ values, when $V_{\mathrm{FG}}=0 \mathrm{~V}$. b Voltage sensitivity and density of inversion carriers $\left(N_{\text {inv }}\right)$ in the bottom channel against back-gate voltage.

interface are shallower and of lower density, with minimal effects on the $\mathrm{n}$-channel region. The donor traps in $\mathrm{WSe}_{2}-\mathrm{MoS}_{2}$ interface effectively weaken or screen the controlling of the $\mathrm{WSe}_{2}$ channel charges by the applied back gate voltage. In addition, the inversion charges that form in the bottom $\mathrm{MoS}_{2}$ layer do not contribute significantly to device current (due to band offset barriers) but can again weaken the back gate control by screening.

To illustrate the effects of screening, parameters such as electric field and charge are plotted against the back gate and fluid gate voltages when the other is set to $0 \mathrm{~V}$ (Fig. 4(c-e)). Figure 4c shows the magnitude of the electric field across the $\mathrm{WSe}_{2}$ channel, which is an order of magnitude lower with the back gate bias than with the applied fluid-gate voltage. The distribution of the electric field is illustrated in Fig. $4 \mathrm{~d}$ for $V_{\mathrm{BG}}=-3 \mathrm{~V}\left(V_{\mathrm{FG}}=0 \mathrm{~V}\right)$ and $V_{\mathrm{FG}}=-3 \mathrm{~V}$ $\left(V_{\mathrm{BG}}=0 \mathrm{~V}\right)$. With the back-gate voltage, the field lines are mainly confined within $\mathrm{MoS}_{2}$, whereas with the applied fluid-gate voltage, there is much higher field penetration. This is evident by considering the electric field direction in the respective channels. Even at $V_{\mathrm{BG}}=-3 \mathrm{~V}\left(V_{\mathrm{FG}}=0 \mathrm{~V}\right)$, the field in the $\mathrm{WSe}_{2}$ channel is directed away from the back gate, i.e., there is a certain amount of band pinning at the $\mathrm{WSe}_{2}-\mathrm{MoS}_{2}$ interface due to the interface traps. However, this does not occur when $V_{\mathrm{FG}}=-3 \mathrm{~V}$ $\left(V_{\mathrm{BG}}=0 \mathrm{~V}\right)$. These effects are also visible in the charge induced in the $\mathrm{WSe}_{2}$ channel by the respective gates, which is shown in Fig. 4 e. The channel charge in $\mathrm{WSe}_{2}$ induced by the back-gate bias is much lower than the channel charge formed by the fluid-gate for a considerable range of voltages. These voltages correspond to the range in $V_{B G}$ where the super-Nernstian response is visible. These factors emphasize the role of screening in reducing the back-gate coupling and the necessity of interface and inversion charges to cause it. This reduced back-gate coupling and hence reduced $g_{m_{b}}$ directly translates to the high sensitivity in our heterostructure-based ISFET.

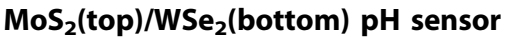

Next, we simulate the flipped $\mathrm{MoS}_{2}$ (top)/WSe $($ bottom) heterostructure device, using the calibrated TCAD model, with $\mathrm{MoS}_{2}$ (ntype) stacked on top of $\mathrm{WSe}_{2}$ (p-type). To be consistent with the simulation setup for $\mathrm{WSe}_{2}$ (top)/ $\mathrm{MoS}_{2}$ (bottom) ISFET, the same donor trap density has been used, while the acceptor traps are being ignored. Although, in a real device, the presence of acceptor traps will reduce the current in the n-region, having an identical simulation setting provides a fair comparison between the

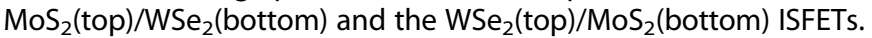
Figure $5 \mathrm{a}$ shows the simulated drain current of the flipped $\mathrm{MoS}_{2}$ (top)/WSe $\mathrm{W}_{2}$ (bottom) ISFET, plotted against $V_{\mathrm{BG}}$ for different $\mathrm{pH}$ values at $V_{\mathrm{FG}}=0 \mathrm{~V}$. Compared with the $\mathrm{WSe}_{2}$ (top)/MoS (bottom) ISFET, the drain current obtained is higher in the $\mathrm{MoS}_{2}$ (top)/ $\mathrm{WSe}_{2}$ (bottom) ISFET. This is because of the lower Schottky barrier height in $\mathrm{MoS}_{2}$ and the absence of acceptor traps in the interface in the simulation setup. The $S_{v}$ of the simulated $M_{0} S_{2}$ (top)/ WSe $\mathrm{S}_{2}$ (bottom) device, shown in Fig. $5 \mathrm{~b}$, displays super-Nernstian sensitivity when the n-layer is ON. On the contrary, the sensitivity remains within the Nernst limit in the p-region. The density of inversion electrons $\left(N_{\text {inv }}\right)$ in the WSe $\mathrm{W}_{2}$ layer, with change in $V_{\mathrm{BG}}$, is also displayed in Fig. 5b. A higher $N_{\text {inv }}$ corresponds to an enhanced $S_{V}$, confirming the contribution of the screening effect in improving sensor performance. Mobility degradation due to higher vertical field at higher $V_{B G}$ may also play a role in enhancing $S_{V}$.

In summary, we have demonstrated a double gated superNernstian ISFET for $\mathrm{pH}$ sensing, using a 2D heterostructure of WSe ${ }_{2}$ (top)/ $\mathrm{MoS}_{2}$ (bottom) as the channel material. The proposed device shows a maximum sensitivity of $362 \mathrm{mV} \mathrm{pH}^{-1}$ for $\mathrm{pH}$ sensing due to the reduced back-gate transconductance. The novelty of this work lies in the underlying sensing mechanism that is attributed to the traps and defects formed at the heterointerface. The bottom semiconducting channel acts both as a screening layer and a site for interface traps, contributing to the enhanced sensitivity. The proposed device and its flipped variant $\left(\mathrm{MoS}_{2}\right.$ (top)/WSe $\mathrm{W}_{2}$ (bottom)) both offer high sensitivity with great tunability, depending on the region of operation (subthreshold, linear, and saturation). Although the Nernst limit can be surpassed in other dual-gate ISFETs by tailoring the thickness and the material of the top and bottom gate dielectrics, the thick back gate oxide limits device scaling. The proposed device, on the other hand, with thin high-k gate oxides and atomically thin 2-D channel materials, offers extreme scalability, enabling ultrascaled, next-generation biosensing platforms for point-of-care applications.

\section{METHODS}

\section{Device fabrication and material characterization}

In all, 30-nm-thick $\mathrm{HfO}_{2}$ was deposited (e-beam evaporation) on $\mathrm{p}++\mathrm{Si}$ substrate, which is used as a global back gate. The $\mathrm{HfO}_{2}$ thin film deposition parameters were optimized based on the reports by Ganapathi et al. $^{38}$. $\mathrm{MoS}_{2}$ flakes (source: Graphene supermarket) were mechanically exfoliated onto this substrate using scotch-tape and polydimethylsiloxane (PDMS) stamps. The samples were cleaned with acetone, isopropyl alcohol (IPA), and deionized water after each process. Flakes, ranging between 4 and $7 \mathrm{~nm}$ in thickness, were identified optically and later confirmed using AFM (Bruker) (Supplementary Fig. 4). Source/drain contacts with a channel length of $1 \mu \mathrm{m}$ were patterned using e-beam lithography (Raith Pioneer), and nickel/palladium (nickel in contact with $\mathrm{MoS}_{2}$ ) of thickness $15 \mathrm{~nm}$ each were deposited using e-beam evaporation, followed by liftoff. WSe $\mathrm{S}_{2}$ flakes (source: 2D Semiconductors) of 4-7 nm thickness were transferred on top of the $\mathrm{MoS}_{2}$ flake using PDMS stamps after aligning them using the stage of an optical lithography setup (MJB4 mask aligner). Source/drain contacts of palladium/gold, each with a thickness of $15 \mathrm{~nm}$, were 
connected to the $\mathrm{WSe}_{2}$ layer, aligned with the contacts on $\mathrm{MoS}_{2}$. The structural and chemical integrity of the flakes were verified using Raman spectroscopy (Supplementary Fig. 4). $\mathrm{HfO}_{2}(\sim 30 \mathrm{~nm})$ was deposited on top, forming the top dielectric, which acts as the $\mathrm{pH}$ sensing layer. This layer also isolates the electrodes from direct contact with the electrolyte. Oxide on the contact pads was etched using buffered hydrofluoric acid (BHF) solution. For the cross-section HR-TEM of the device, the sample was prepared using focused ion beam (FIB) Helios G4-UX and imaged using Titan Themis $300 \mathrm{kV}$ from ThermoFisher.

\section{Device characterization}

PBS was used as the electrolyte. PBS solution with different $\mathrm{pH}$ values, ranging from $\mathrm{pH}=5$ to 8 , was prepared and the $\mathrm{pH}$ was accurately determined using a standard laboratory $\mathrm{pH}$ meter (Eutech Instruments). The electrolyte solution is then drop-casted $(\sim 10 \mu \mathrm{l})$ onto the exposed region (top $\mathrm{HfO}_{2}$ layer) of the ISFET for sensing. A fluid gate, made up of a platinum electrode, connects to the top $\mathrm{HfO}_{2}$ layer through the electrolyte solution, and is used to maintain a constant reference potential during measurement. Finally, the electrical measurements were carried out using Keysight B1500 Semiconductor device analyzer at room temperature.

\section{TCAD simulations}

The current-voltage characteristics of $\mathrm{WSe}_{2}$ (top)/ $\mathrm{MoS}_{2}$ (bottom) and $\mathrm{MoS}_{2}$ (top)/WSe (bottom) ISFET heterostructure devices were simulated using Silvaco ATLAS simulator ${ }^{51}$. Material parameters of $\mathrm{MoS}_{2}$ and $\mathrm{WSe}_{2}$, such as carrier mobility, were defined based on the reports by Mirabelle et al. ${ }^{47,48}$ and Arora et al. ${ }^{52}$. Shockley-Read-Hall (SRH) and Auger models $s^{53,54}$ for carrier generation-recombination were also included. Quantum effects have been ignored in the TCAD to simplify the simulation procedure. The electrolyte was modeled using an undoped semiconductor. The details of this modeling are given in Supplementary Notes.

The simulated ISFET was then calibrated against the experiment-transfer curve, corresponding to $\mathrm{pH}=7$. The device parameters such as mobility, doping, interface traps, and Schottky barrier heights were adjusted in the TCAD model to match the experimental results accurately. These final values after calibration of these parameters are provided in Supplementary Table 2, along with the material parameters used in the simulation. To better fit the subthreshold region of the transfer curve and its shape, interface traps and fixed-oxide charges were added at various interfaces. These are summarized in Table 3 of the Supplementary Information.

\section{DATA AVAILABILITY}

The data that support the findings of this study are available from the corresponding author upon reasonable request.

Received: 20 June 2021; Accepted: 29 October 2021; Published online: 16 December 2021

\section{REFERENCES}

1. Fakih, I. et al. Selective ion sensing with high resolution large area graphene field effect transistor arrays. Nat. Commun. 11, 1-12 (2020).

2. Lim, D. V., Simpson, J. M., Kearns, E. A. \& Kramer, M. F. Current and developing technologies for monitoring agents of bioterrorism and biowarfare. Clin. Microbiol. Rev. 18, 583-607 (2005).

3. Sadighbayan, D., Hasanzadeh, M. \& Ghafar-Zadeh, E. Biosensing based on fieldeffect transistors (FET): Recent progress and challenges. TrAC Trends Anal. Chem. 133, 116067 (2020).

4. Syu, Y. C., Hsu, W. E. \& Lin, C. T. Review-Field-effect transistor biosensing: devices and clinical applications. ECS J. Solid State Sci. Technol. 7, Q3196-Q3207 (2018).

5. Keeble, L., Moser, N., Rodriguez-Manzano, J. \& Georgiou, P. ISFET-based sensing and electric field actuation of DNA for on-chip detection: a review. IEEE Sens. J. 20, 11044-11065 (2020).

6. Kaisti, M. et al. An ion-sensitive floating gate FET model: operating principles and electrofluidic gating. IEEE Trans. Electron Devices 62, 2628-2635 (2015).

7. Chen, S., Bomer, J. G., Carlen, E. T. \& Van Den Berg, A. $\mathrm{Al}_{2} \mathrm{O}_{3} /$ silicon nanolSFET with near ideal nernstian response. Nano Lett. 11, 2334-2341 (2011).

8. Bergveld, P. Thirty years of ISFETOLOGY: what happened in the past 30 years and what may happen in the next 30 years. Sens. Actuators B Chem. 88, 1-20 (2003).
9. Spijkman, M. et al. Beyond the Nernst-limit with dual-gate $\mathrm{ZnO}$ ion-sensitive fieldeffect transistors. Appl. Phys. Lett. 98, 2011-2014 (2011).

10. Bellando, F. et al. Subthermionic negative capacitance ion sensitive field-effect transistor. Appl. Phys. Lett. 116, 173503 (2020).

11. Dwivedi, P., Singh, R. \& Chauhan, Y. S. Crossing the Nernst Limit $(59 \mathrm{mV} / \mathrm{pH})$ of sensitivity through tunneling transistor-based biosensor. IEEE Sens. J. 21, 3233-3240 (2021).

12. Knopfmacher, O. et al. Nernst limit in dual-gated Si-nanowire FET sensors. Nano Lett. 10, 2268-2274 (2010).

13. $\mathrm{Wu}, \mathrm{T}$. et al. Experimental study of the detection limit in dual-gate biosensors using ultrathin silicon transistors. ACS Nano 11, 7142-7147 (2017).

14. Go, J. et al. Beating the Nernst limit of $59 \mathrm{mV} / \mathrm{pH}$ with double-gated nano-scale field-effect transistors and its applications to ultra-sensitive DNA biosensors. in IEDM Technical Digest. 8.7.1-8.7.4 (IEEE, 2010).

15. Lu, C., Hou, T. \& Pan, T. High-Performance Double-Gate alpha-InGaZnO ISFET pH sensor Using a HfO2 Gate dielectric. IEEE Trans. Electron Devices 65, 237-242 (2018).

16. Jeon, J. H. \& Cho, W. J. Ultrasensitive coplanar Dual-Gate ISFETs for point-of-care biomedical applications. ACS Omega 5, 12809-12815 (2020).

17. Nair, P. R. \& Alam, M. A. Screening-limited response of NanoBiosensors. Nano Lett. 8, 1281-1285 (2008).

18. Spijkman, M. J. et al. Dual-gate organic field-effect transistors as potentiometrie sensors in aqueous solution. Adv. Funct. Mater. 20, 898-905 (2010).

19. Kalantar-Zadeh, K. \& Ou, J. Z. Biosensors based on two-dimensional MoS 2 . ACS Sens. 1, 5-16 (2016).

20. Sarkar, D. et al. MoS , field-effect transistor for next-generation label-free biosensors. ACS Nano 8, 3992-4003 (2014).

21. Nam, H. et al. Fabrication and comparison of $\mathrm{MoS}_{2}$ and $\mathrm{WSe}_{2}$ field-effect transistor biosensors. J. Vac. Sci. Technol. B 33, 06FG01 (2015).

22. Wang, H., Zhao, P., Zeng, X., Young, C. D. \& Hu, W. High-stability pH sensing with a few-layer $\mathrm{MoS}_{2}$ field-effect transistor. Nanotechnology 30, 375203 (2019).

23. Lee, J. et al. Two-dimensional layered $\mathrm{MoS}_{2}$ biosensors enable highly sensitive detection of biomolecules. Sci. Rep. 4, 1-7 (2014).

24. Zagni, N., Pavan, P. \& Alam, M. A. Two-dimensional $\mathrm{MoS}_{2}$ negative capacitor transistors for enhanced (super-Nernstian) signal-to-noise performance of nextgeneration nano biosensors. Appl. Phys. Lett. 114, 233102 (2019).

25. Jeon, H. B., Shin, G. H., Lee, K. J. \& Choi, S. Y. Vertical-tunneling field-effect transistor based on $\mathrm{WSe}_{2}-\mathrm{MoS}_{2}$ heterostructure with ion gel dielectric. Adv. Electron. Mater. 6, 1-6 (2020)

26. $\mathrm{Wu}, \mathrm{D}$. et al. Visualization of local conductance in $\mathrm{MoS}_{2} / \mathrm{WSe}_{2}$ heterostructure transistors. Nano Lett. 19, 1976-1981 (2019).

27. $\mathrm{Li}, \mathrm{C}$. et al. $\mathrm{WSe}_{2} / \mathrm{MoS}_{2}$ and $\mathrm{MoTe}_{2} / \mathrm{SnSe}_{2}$ van der Waals heterostructure transistors with different band alignment. Nanotechnology 28, 415201 (2017).

28. Nourbakhsh, A., Zubair, A., Dresselhaus, M. S. \& Palacios, T. Transport properties of a $\mathrm{MoS}_{2} / \mathrm{WSe}_{2}$ heterojunction transistor and its potential for application. Nano Lett. 16, 1359-1366 (2016).

29. Wang, $\mathrm{H}$. et al. Two-dimensional heterostructures: fabrication, characterization, and application. Nanoscale 6, 12250-12272 (2014).

30. Fu, W., Jiang, L., Geest, E. P., van, Lima, L. M. C. \& Schneider, G. F. Sensing at the surface of graphene field-effect transistors. Adv. Mater. 29, 1603610 (2017).

31. Jung, S.-H. et al. Super-Nernstian $\mathrm{pH}$ sensor based on anomalous charge transfer doping of defect-engineered graphene. Nano Lett. 21, 34-42 (2020).

32. Lee, C. W. et al. Surface-tailored graphene channels. npj 2D Mater. Appl. 2021 1-13 (2021). 515

33. Van Der Wal, P. D. et al. High-k dielectrics for use as ISFET gate oxides. Proc. IEEE Sens. 2, 677-680 (2004).

34. Lai, C. S., Yang, C. M. \& Lu, T. F. pH sensitivity improvement on $8 \mathrm{~nm}$ thick hafnium oxide by post deposition annealing. Electrochem. Solid State Lett. 9 90-93 (2006).

35. Fredj, Z. et al. Capacitance electrochemical $\mathrm{pH}$ sensor based on different hafnium dioxide $\left(\mathrm{HfO}_{2}\right)$ thicknesses. Chemosensors 9, 1-13 (2021).

36. Ganapathi, K. L., Bhattacharjee, S., Mohan, S. \& Bhat, N. High-performance $\mathrm{HfO}_{2}$ back gated multilayer $\mathrm{MoS}_{2}$ transistors. IEEE Electron Device Lett. 37, 797-800 (2016).

37. Smyth, C. M. et al. Engineering the palladium $-W_{S e_{2}}$ interface chemistry for field effect transistors with high-performance hole contacts. ACS Appl. Nano Mater. 2, 75-88 (2019).

38. Lakshmi Ganapathi, K., Bhat, N. \& Mohan, S. Optimization and integration of ultrathin e-beam grown $\mathrm{HfO}_{2}$ gate dielectrics in $\mathrm{MoS}_{2}$ transistors. J. Phys. D Appl. Phys. 54, 445302 (2021)

39. Lee, I. et al. Gate-tunable hole and electron carrier transport in atomically thin dual-channel $\mathrm{WSe}_{2} / \mathrm{MoS}_{2}$ heterostructure for ambipolar field-effect transistors. Adv. Mater. 28, 9519-9525 (2016).

40. Kaushik, N. et al. Reversible hysteresis inversion in $\mathrm{MoS}_{2}$ field effect transistors. npj 2D Mater. Appl. 1, 1-9 (2017). 
41. Knobloch, T. et al. A physical model for the hysteresis in $\mathrm{MoS}_{2}$ transistors. IEEE J. Electron Devices Soc. 6, 972-978 (2018).

42. Go, J., Nair, P. R. \& Alam, M. A. Theory of signal and noise in double-gated nanoscale electronic pH sensors. J. Appl. Phys. 112, 034516 (2012).

43. Shadman, A., Rahman, E. \& Khosru, Q. D. M. Monolayer $\mathrm{MoS}_{2}$ and $\mathrm{WSe}_{2}$ double gate field effect transistor as super Nernst $\mathrm{pH}$ sensor and nanobiosensor. Sens. BioSens. Res. 11, 45-51 (2016).

44. Jang, H. J. \& Cho, W. J. Fabrication of high-performance fully depleted silicon-oninsulator based dual-gate ion-sensitive field-effect transistor beyond the Nernstian limit. Appl. Phys. Lett. 100, 073701 (2012).

45. Koo, J., Gao, S., Lee, H. \& Yang, L. Vertical dielectric screening of few-layer van der Waals semiconductors. Nanoscale 9, 14540-14547 (2017).

46. Mori, T. et al. Characterization of effective mobility and its degradation mechanism in $\mathrm{MoS}_{2}$ MOSFETs. IEEE Trans. Nanotechnol. 15, 651-656 (2016).

47. Mirabelli, G., Gity, F., Monaghan, S., Hurley, P. K. \& Duffy, R. Impact of impurities, interface traps and contacts on $\mathrm{MoS}_{2}$ MOSFETs: modelling and experiments. European Solid State Device Research Conference, 288-291 (IEEE, 2017)

48. Mirabelli, G. Two-dimensional semiconductors for future electronics, PhD Thesis, University College Cork. (2020)

49. Yang, G. W., Seo, S. G., Choi, S., Kim, D. H. \& Jin, S. H. Unscrambling for subgap density-of-states in multilayered $\mathrm{MoS}_{2}$ field effect transistors under DC bias stress via optical charge-pumping capacitance-voltage spectroscopy. IEEE Access 9, 73090-73102 (2021).

50. Mohammadi, E. \& Manavizadeh, N. An Accurate TCAD-based model for ISFET simulation. IEEE Trans. Electron Devices 65, 3950-3956 (2018).

51. Silvaco Inc. ATLAS User's Manual: Device Simulation Software. Silvaco Int., Santa Clara, CA (2016).

52. Arora, N. D., Hauser, J. R. \& Roulston, D. J. Electron and hole mobilities in silicon as a function of concentration and temperature. IEEE Trans. Electron Devices 29, 292-295 (1982).

53. Lee, M. H. et al. Ferroelectric negative capacitance hetero-tunnel field-effecttransistors with internal voltage amplification. in IEDM Technical Digest. 4.5.1-4.5.4 (IEEE, 2013).

54. Liu, C. et al. Simulation-based study of negative-capacitance double-gate tunnel field-effect transistor with ferroelectric gate stack. Jpn. J. Appl. Phys. 55, 8-12 (2016).

\section{ACKNOWLEDGEMENTS}

The authors acknowledge the technical support provided by staff at National Nanofabrication Centre (NNFC), Micro and Nano Characterization Facility (MNCF) at the Centre for Nano Science and Engineering (CeNSE), Indian Institute of Science, Bengaluru, India. The authors also acknowledge technical support from the Advanced Facility for Microscopy and Microanalysis (AFMM) at Indian Institute of Science, Bengaluru, India. The authors would also like to thank Dr. K. Lakshmi Ganapathi (Indian Institute of Technology Madras, Chennai, India) and Dr. S. Dawnee (Ramaiah Institute of Technology, Bengaluru, India) for useful discussions. This work was supported by the Ministry of Human Resource Development (MHRD), Department of Electronics and Information Technology (DeitY), and Department of Science and
Technology (DST) Nano Mission through Nanoelectronics Network for Research and Application (NNetRA). The authors also acknowledge the funding support from the Department of Science and Technology (Government of India) under India Science and Research Fellowship (ISRF) program.

\section{AUTHOR CONTRIBUTIONS}

N. Bhat conceived the project. S. Sanjay and M. Hossain fabricated the devices, performed the characterizations, and conducted the experiments. A. Rao carried out TEM sample preparation, characterization, and analysis. S. Sanjay carried out the TCAD simulations with inputs from M. Hossain. S. Sanjay and M. Hossain analyzed the results and prepared the paper with $\mathrm{N}$. Bhat. All authors approve the final version of the paper.

\section{COMPETING INTERESTS}

The authors declare no competing interests.

\section{ADDITIONAL INFORMATION}

Supplementary information The online version contains supplementary material available at https://doi.org/10.1038/s41699-021-00273-6.

Correspondence and requests for materials should be addressed to Navakanta Bhat.

Reprints and permission information is available at http://www.nature.com/ reprints

Publisher's note Springer Nature remains neutral with regard to jurisdictional claims in published maps and institutional affiliations.

Open Access This article is licensed under a Creative Commons Attribution 4.0 International License, which permits use, sharing, adaptation, distribution and reproduction in any medium or format, as long as you give appropriate credit to the original author(s) and the source, provide a link to the Creative Commons license, and indicate if changes were made. The images or other third party material in this article are included in the article's Creative Commons license, unless indicated otherwise in a credit line to the material. If material is not included in the article's Creative Commons license and your intended use is not permitted by statutory regulation or exceeds the permitted use, you will need to obtain permission directly from the copyright holder. To view a copy of this license, visit http://creativecommons. org/licenses/by/4.0/.

(c) The Author(s) 2021 